\title{
Construction of Epidermal Growth Factor Receptor Peptide Magnetic Nanovesicles with Lipid Bilayers for Enhanced Capture of Liver Cancer Circulating Tumor Cells
}

Jian Ding ${ }^{1, \#, *}$, Kai Wang ${ }^{2, \#}$, Wen-Jie Tang ${ }^{3, *}$, Dan $\mathrm{Li}^{4}$, You-Zhen Wei ${ }^{3}$, Ying $\mathrm{Lu}^{3}$, Zong-Hai $\mathrm{Li}^{2}$ and Xiao-Fei Liang, ${ }^{2, *}$

${ }^{1}$ Digestive Department, the First Affiliated Hospital of Fujian Medical University, 20 Chazhong Road, Fuzhou 350005, China.

2 State Key Laboratory of Oncogenes and Related Genes, Shanghai Cancer Institute, Renji Hospital, Shanghai Jiaotong University School of Medicine, No.25/Ln2200 Xie Tu Road, Shanghai 200032, China.

3 Research Centre for Translational Medicine, East Hospital, Tongji University School of Medicine, 150 Jimo Road Shanghai 200120, China.

${ }^{4}$ Digestive Department, Union Hospital of Fujian Medical University, Fuzhou 350001, China.

\# These authors contributed equally to this paper.

*Co-corresponding authors: Jian Ding, Email: docdingjian@163.com, Fax:+8659188808826 ; Wen-Jie Tang, E-mail: wenjie_tang@163.com; Xiao-Fei Liang E-mail: xfliang@shsci.org 
Table S1. Solubility and zeta potential of GE11-GHDC with different degrees of quaternary substitution (DS).

\begin{tabular}{|c|c|c|c|c|c|c|c|c|c|}
\hline \multirow{2}{*}{ Sample } & \multirow[b]{2}{*}{ DS (\%) } & \multirow{2}{*}{$\zeta$ potential $(\mathrm{mV})$} & \multicolumn{7}{|c|}{ Solubility } \\
\hline & & & $\mathrm{H}_{2} \mathrm{O}$ & DMSO & THF & $\mathrm{CHCl}_{3}$ & Toluene & Acetone & $1 \% \mathrm{HAc}$ \\
\hline GE11 peptide & 0.0 & $+37.8 \pm 13.0$ & + & - & - & - & - & - & ++ \\
\hline GHDC & 100.0 & --- & + & + & + & + & + & + & ++ \\
\hline GE11-GHDC 1 & $9.1 \%$ & $+49.5 \pm 3.9$ & + & - & - & - & - & - & ++ \\
\hline GE11-GHDC 2 & $38.8 \%$ & $+43.1 \pm 3.4$ & + & - & - & - & - & - & ++ \\
\hline GE11-GHDC 3 & $75.8 \%$ & $+50.1 \pm 3.9$ & + & + & + & ++ & + & - & ++ \\
\hline GE11-GHDC 4 & $90.8 \%$ & $+53.7 \pm 4.2$ & + & + & + & + & + & - & ++ \\
\hline
\end{tabular}

++ , highly Soluble; + , Soluble; \pm , partially soluble or swelled; - , insoluble. 

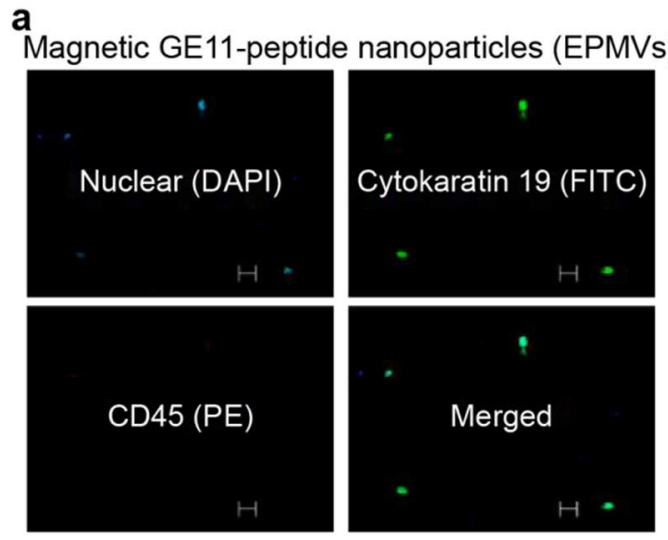

b EpCAM magnetic nanobeads in the
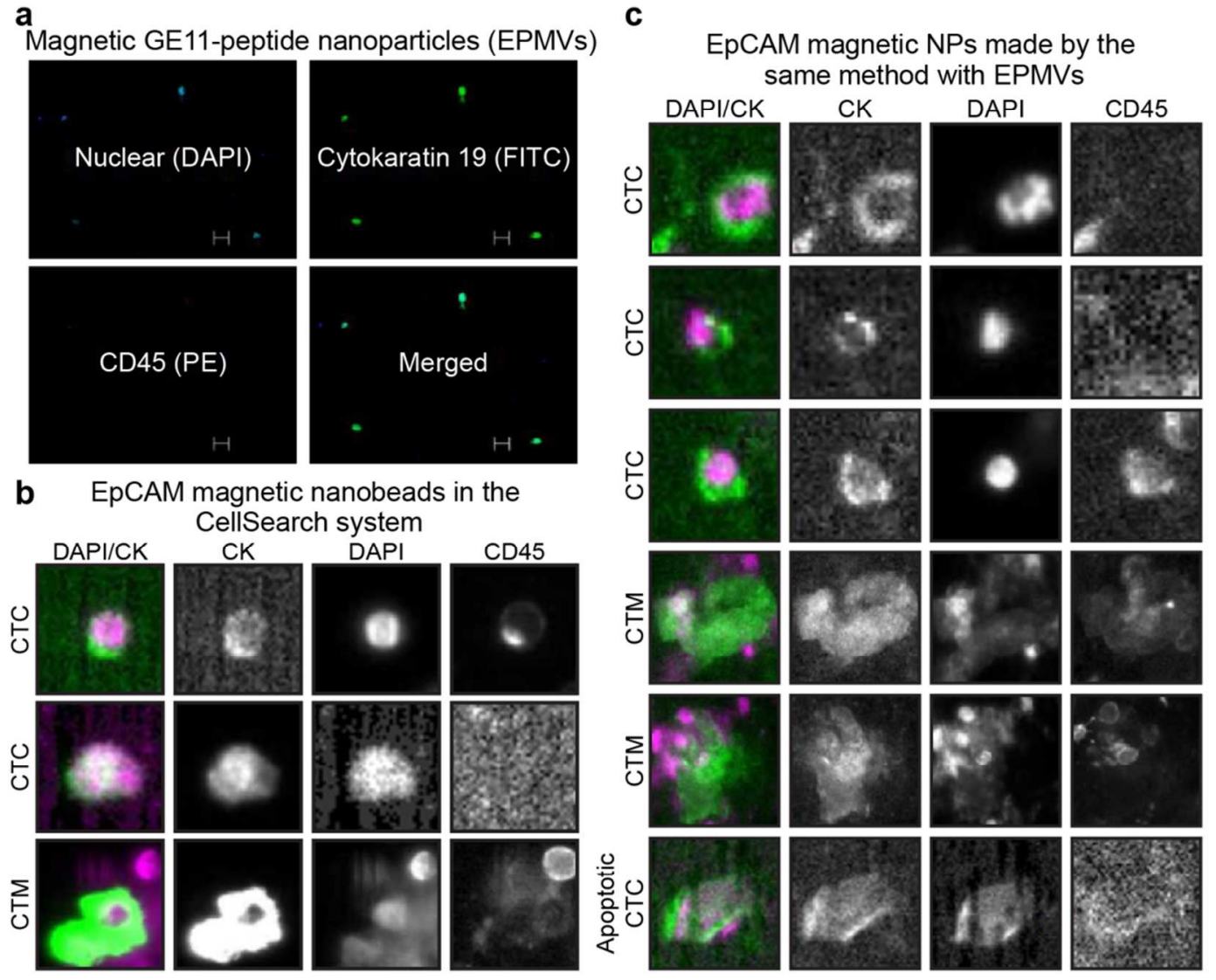

Figure S1. Typical images of EGFR ${ }^{+}$CTCs identified with EPMVs by fluorescence microscope (a), EpCAM magnetic nanobeads in CellSearch system (b) and EpCAM magnetic NPs made by the same method with EPMVs (c) with the CellSearch system. CTCs are defined as cells staining positively for $\mathrm{CK}$ and DAPI and negatively for CD45. Circulating tumor microemboli (CTM) are defined as CTC clusters. Apoptotic CTCs are defined as CTCs with fragmented, condensed DAPI-stained nuclei morphology. 